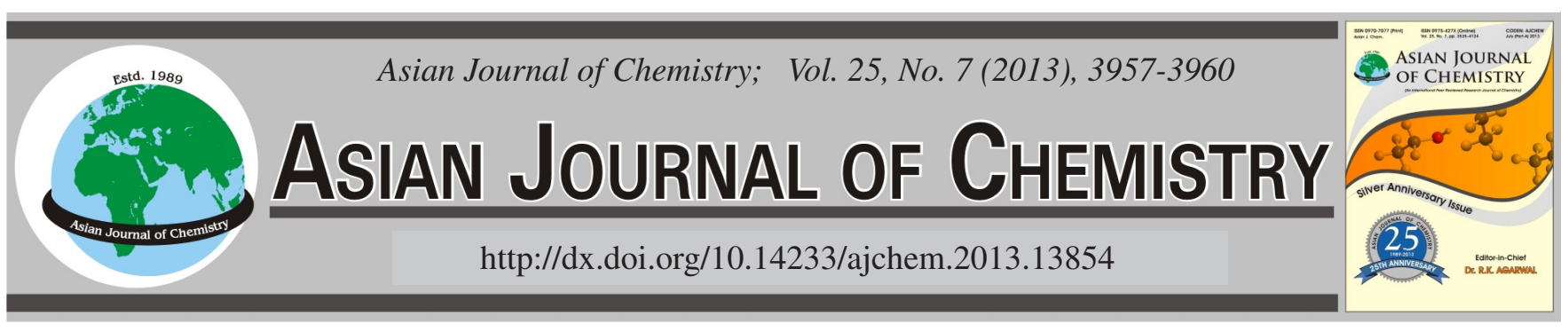

\title{
Antimicrobial and Antitumor Activities of Crude Extracts and Isolated Compounds from Euphorbia humifusa
}

\author{
Jianbo Lin, Haiging Zhao, Ba Hang and Haji Akber Aisa*
}

Key Laboratory of Chemistry of Plant Resources in Arid Regions, Xinjiang Technical Institute of Physics and Chemistry, Chinese Academy of Sciences, Urumqi, P.R. China

*Corresponding author: Fax: +86 991 3838957; Tel: +86 991 3835679; E-mail: haji@ms.xjb.ac.cn

(Received: 6 April 2012;

Accepted: 18 January 2013)

AJC-12731

\begin{abstract}
Euphorbia humifusa Willd. has been used in traditional Chinese medicine. Herein we report the isolation of its constituents and the testing results of their antimicrobial and antitumor activities. The results show that the crude petroleum ether, chloroform, ethyl acetate, butanol and residual methanol fractions exert such activities. Particularly, the crude petroleum ether and ethyl acetate fractions exhibit antigrowth activity against Staphyloccocus aureus with MIC $0.5 \mathrm{mg} / \mathrm{mL}$. Petroleum ether and chloroform fractions significantly exhibit potent antigrowth activity against Caco-2 cells, with $\mathrm{IC}_{50}$ value of 0.059 and $0.025 \mathrm{mg} / \mathrm{mL}$, respectively. The promising bioactivities of the crude petroleum ether fractions guided the first isolation of compounds: 24-methylene cycloartanone (2), dibutyl phthalate (3), hexacosanol (4) and bis-(2-ethylhexyl) phthalate (5) from this species. The antimicrobial activities of compounds 1-2 were evaluated. The tested compounds exhibit potent antigrowth activity against Staphyloccocus aureus, Escherichia coli and Canidia albicans.
\end{abstract}

Key Words: Euphorbia humifusa, Ethyl acetate extract, Antimicrobial activity, Antitumor activity.

\section{INTRODUCTION}

Natural products which were derived from plants have been widely used in medicine since they own pharmacological activities such as antibacterial, antifungal, antiviral, antiparasitic, insecticidal and antispasmodic ${ }^{1-5}$. Today they are being used in pharmaceutical, sanitary, cosmetic, agricultural and food industries.

Euphorbia genus belongs to the family Euphorbiaceae. This family includes 300 genera and over 5000 species ranging from annuals to trees ${ }^{6}$. Many species of the genus Euphorbia have been used as medicinal plants for the treatment of skin diseases, gonorrhea, migraine and intestinal parasites and as wart cures ${ }^{7}$.

The widespread plants of the genus Euphorbia (Euphorbiaceae) are rich in biologically active compounds with diverse structures. For example, pubescenol, helioscopinolide A, helioscopinolide B and pubescene D, isolated from E. pubescens, were shown to be moderate inhibitors of the human tumor cell lines MCF-7 (breast), NCI H460 (lung) and SF-268 (central nervous system). Moreover, helioscopinolide A and helioscopinolide B showed significant activity against Staphylococcus aureus 6538P $(2.5 \mu \mathrm{g} / \mathrm{spot})^{8}$. Jolkinolide A from $E$. sessiliflora showed moderate growth inhibition against M. catarrhalis at $50 \mu \mathrm{g} / \mathrm{mL}$ concentration ${ }^{9}$. A three jatrophane diterpenoids isolated from E. mongolica was found to show a concentration-dependent effect in inhibiting the efflux pump activity of the tumor cells in the range $11.2-112 \mu \mathrm{M}^{10}$. Natarajan et al. ${ }^{11}$ reported that the different extracts of $E$. fusiformis differed significantly in their antibacterial properties. Methanol extract comparing with acetone and chloroform extracts was very effective. Especially, rootstock extracts had better antibacterial properties than leaf extracts. In addition, the ethanol extracts of aerial parts of $E$. hirta also exhibited broad spectrum of antimicrobial activity against E. coli, P. Vulgaris, P. aeruginosa and $S$. aureus $^{12}$.

As an important plant of the family Euphorbia, Euphorbia humifusa Willd has been used in traditional Chinese medicine for the treatment of dysentery, enteritis and hematochezia ${ }^{13}$. It has been reported that three new hydrolyzable tannins have been isolated from $E$. humifusa ${ }^{14}$. Lu et al. ${ }^{15}$ have obtained four new lanostane triterpenoids from $E$. humifusa.

However, previous studies of E. humifusa were focused on the chemical constituents and few chemical isolation and characterization of the components of this medicinal plant have been reported. Hence, the present study attempts to evaluate its in vitro antimicrobial and antitumor activities so as to support ethnopharmacological relevance of the plant. In addition, the study also assessed the chemical constituents of E. humifusa. 


\section{EXPERIMENTAL}

The total plant of E. humifusa was collected in 2008 from the Burqin region of Xinjiang (Northwest China) and initially identified by Dr. G.M. Shen, Xinjiang Institute of Ecology and Geography and a voucher specimen has been deposited in Xinjiang Technical Institute of Physics and Chemistry with voucher number A0009.

Preparation of crude extracts: The air-dried plant materials were pulverized into powdered form. The dried powder $(10 \mathrm{Kg})$ was extracted three times with methanol $(3 \times$ $75 \mathrm{~L}$ ) at room temperature and the solvent from the combined extracts were evaporated by a vacuum rotary evaporator (BUCHI R-210, Switzerland). The methanol extract (880 g) was suspended in water and extracted successively with petroleum ether, chloroform, ethyl acetate and butanol to give petroleum ether $(270 \mathrm{~g})$, chloroform $(90 \mathrm{~g})$, ethyl acetate (60 $\mathrm{g})$, butanol $(210 \mathrm{~g})$ and residual methanol fractions $(220 \mathrm{~g})$, respectively.

Isolation and purification: The crude petroleum ether extract (75 g) was loaded on a silica gel column (200-300 mesh) and eluted with petroleum-ether containing increasing amounts of acetone to afford compounds 1-8 respectively.

Antitumor activity: Caco-2 cells (human colourectal carcinoma) were obtained from the Cell Bank of the Chinese Academy of Sciences (Shanghai, China). Cells were maintained in Dulbecco's modified Eagle's medium (DMEM). When the cells were 80-90 \% confluent, they were harvested by treatment with a solution containing $0.25 \%$ trypsin, thoroughly washed and resuspended in supplemented growth medium. Cells were inoculated into 96-well microtiter plates in $100 \mu \mathrm{L}$ at plating densities ranging $1.0 \times 10^{5}$ cells/well depending on the doubling time of individual cell lines. After cell inoculation, the microtiter plates were incubated at $37^{\circ} \mathrm{C}$, $5 \% \mathrm{CO}_{2}, 95 \%$ air and $100 \%$ relative humidity.

The next day, samples were prepared in DMSO and the concentration ranges tested were 250, 25, 2.5, 0.25 and 0.025 $\mu \mathrm{g} / \mathrm{mL}$. The establishment of five groups of three parallel holes, then $1 \mu \mathrm{L}$ of corresponding concentration of sample were added to each well.

MTT assay was employed as test for quantification of cell proliferation after $24 \mathrm{~h}$ incubation. For this purpose, the medium of each well was removed and $50 \mu \mathrm{L}$ of MTT solution $(1 \mathrm{mg} / \mathrm{mL})$ was added. After $4 \mathrm{~h}$, the medium was removed and the cells were washed twice with PBS, then replaced with $150 \mu \mathrm{L}$ of DMSO to dissolve the MTT crystals.

The optical density was determined using a Microplate reader SpectraMax M5 (molecular devices) at a test wavelength of $550 \mathrm{~nm}$. The inhibitory percentage of each sample at various concentrations was calculated and the $\mathrm{IC}_{50}$ value was determined. Each data is the mean value of three determinations.

Antimicrobial activity: Three type strains, Staphyloccocus aureus ATCC 6538, Escherichia coli ATCC 11229, Canidia albicans ATCC 10231 were obtained from the National Center for Medical Culture Collections (CMCC), China. All strains were stored at $-80^{\circ} \mathrm{C}$ in the appropriate medium. Antimicrobial activity of the compounds and crude extract was tested using the agar well diffusion method ${ }^{16}$. Luria-Bertani (LB) and Sabaurauds agar (SDA) were sterilized in an autoclave and cooled to $45-50{ }^{\circ} \mathrm{C}$ before being poured into $100 \mathrm{~mm}$ Petri dishes. The agar plates were stored at $4{ }^{\circ} \mathrm{C}$ before being used.

Staphyloccocus aureus ATCC 6538 and Escherichia coli ATCC 11229 were cultured overnight at $37^{\circ} \mathrm{C}$ in Luria-Bertani. Canidia albicans ATCC 10231 was cultured overnight at $37^{\circ} \mathrm{C}$ in Sabaurauds agar. Petri dishes with $20 \mathrm{~mL}$ of medium were prepared, previously inoculated with $200 \mu \mathrm{L}$ of the culture suspension. The wells $(6 \mathrm{~mm})$ were made and the sample diluted in DMSO to test concentration $(100 \mathrm{mg} / \mathrm{mL})$ was added (20 $\mu \mathrm{L} /$ well) and the same volume $(20 \mu \mathrm{L})$ of DMSO was used as a control. The inoculated plates were incubated for 24/48 h. After incubation, the diameter of the inhibition zone was measured with calipers.

The minimum inhibitory concentration (MIC) values and the minimum fungicidal concentration (MFC)/minimum bactericidal concentration (MBC) values were determined for the microorganisms that were sensitive to the essential oil in the agar well diffusion method.

The minimum inhibitory concentration determination was performed by a serial dilution technique, using 96-well microtitre plates. The bacterial inocula applied contained approximately $1.0 \times 10^{5}$ cells in a final $200 \mu \mathrm{L} /$ well volume. The extracts tested were dissolved in DMSO (0.01-100 mg/ $\mathrm{mL}$ ) and added to medium with microbial inocula. The microplates were incubated for $24 / 48 \mathrm{~h}$ at $37^{\circ} \mathrm{C}$. The lowest concentrations without visible growth (at the binocular microscope) were defined as concentrations which completely inhibited bacterial growth (MIC). The MFC/MBC were determined by serial subcultivation of $4 \mu \mathrm{L}$ in microtitre plates containing $196 \mu \mathrm{L}$ of broth per well and further incubation for 24/48 h at $37^{\circ} \mathrm{C}$. The lowest concentration with no visible growth was defined as the MBC/MFC. DMSO was used as a negative control, while ampicillin and amphotericin $\mathrm{B}$ were used as a positive control. Dilutions of the inocula were also cultured on solid Luria-Bertani and Sabaurauds agar to verify the absence of contamination and to check their validity. Each assay replicated three times.

Statistical analysis: The results were expressed as $\mathrm{IC}_{50}$ values, which were calculated by using Microsoft@ Excel. The statistical analysis of all the observations were carried out using one-way ANOVA followed by multiple comparison test of Tukey-Kramer, where necessary $\mathrm{P}<0.05$ was considered as significant compared with the control.

\section{RESULTS AND DISCUSSION}

Antimicrobial and antitumor activities of crude extracts: Extracts of different polarity (petroleum ether, chloroform, ethyl acetate, butanol and residual methanol fractions) obtained from $E$. humifusa have been investigated for their antimicrobial activity against Staphyloccocus aureus, Canidia Albicans and Escherichia coli. The most potent growth inhibitory action was shown by petroleum ether and ethyl acetate fraction with an $\mathrm{IC}_{50}$ value of $0.5 \mathrm{mg} / \mathrm{mL}$. Staphyloccocus aureus strains showed more sensibility to those extracts when compared with Escherichia coli and Canidia albicans strains. The possible reason was that Gram-positive bacteria appeared to be more sensitive than Gram-negative. Evaluation of minimum inhibitory concentration and MBC/MFC showed that the extracts 
TABLE-1

ANTIMICROBIAL ACTIVITY (MIC, MBC/MFC) OF CRUDE EXTRACTS OF E. humifusa for Staphyloccocus aureus, Escherichia coli and Canidia albicans strains. MIC AND MBC/MFC WERE DETERMINED BY A MACRODILUTION METHOD AND EXPRESSED IN mg/mL

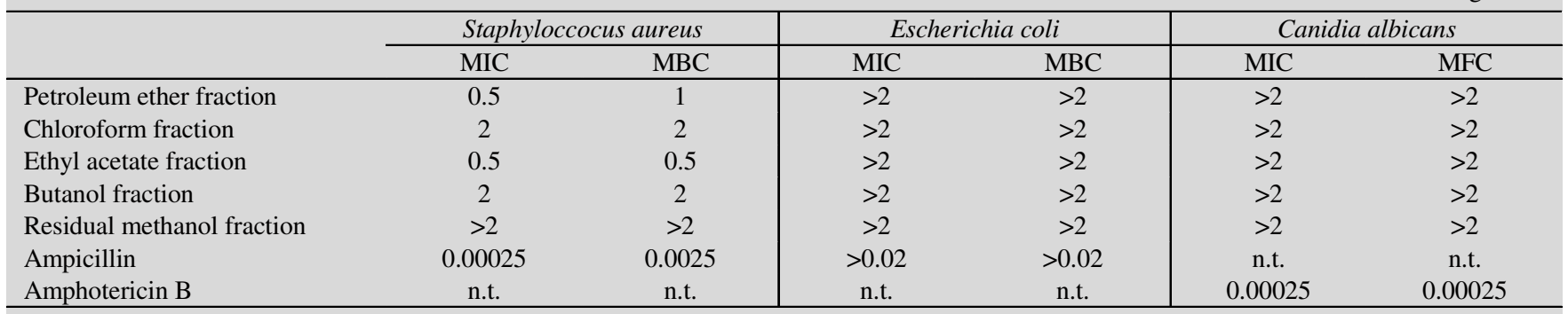

MIC: minimum inhibitory concentration; MFC/MBC: minimum fungicidal/bactericidal concentration; n.t.: not tested

presented various degrees of inhibition against all these microbes investigated (Table-1).

In addition, the study also assessed the antitumor activity of different polarity extracts from E. humifusa. The results show that petroleum ether and chloroform fractions exhibits significant antigrowth activity against Caco- 2 cells, with $\mathrm{IC}_{50}$ value of 0.059 and $0.025 \mathrm{mg} / \mathrm{mL}$, respectively. However, no antitumor activities were observed at ethyl acetate, butanol and residual methanol fractions. There is no report on the antimicrobial and antitumor activities of E. humifusa on the above mentioned strains and human cancer cell lines, except those for the Staphyloccocus aureus.

Isolation and identification of compounds: As the petroleum ether fraction demonstrated potent inhibitory activity, further chemical investigation was focused on this fraction. Chemical investigation from the petroleum ether fractions resulted in the isolation of eight pure compounds, namely cycloart-23E-en-3 $\beta$-25-diol (1), 24-methylene cycloartanone (2), dibutyl phthalate (3), hexacosanol (4), bis-(2-ethylhexyl) phthalate (5), stigmasterol (6), sitosterol (7), hexadecanoic acid (8). All chemical components were identified by comparison of their mass and NMR spectra with published data and found to be in good agreement $t^{17-22}$. The compounds 2-5 are reported here as natural constituents of E. humifusa for the first time.

Antimicrobial activities of the isolated pure compounds: The isolated 1-2 were evaluated for antimicrobial activity against Staphyloccocus aureus, Canidia albicans and Escherichia coli, using gallic acid as a positive control. It was observed that the tested compounds 1-2 (Table-2) showed moderate antimicrobial activities (MIC $=2000 \mu \mathrm{M} / \mathrm{mL})$.

Cycloart-23E-en-3 $\beta$-25-diol (1) was first found in nature from E. petiolata ${ }^{18}$. It was also isolated from E. humifusa ${ }^{17}$. 24-Methylene cycloartanone (2) was reported for the first time

TABLE-2

ANTIMICROBIAL ACTIVITY (MIC, MBC/MFC) OF ISOLATED

COMPOUNDS OF E. humifusa FOR Staphyloccocus aureus,

Escherichia coli AND Canidia albicans strains. MIC AND

MBC/MFC WERE DETERMINED BY A MACRODILUTION METHOD AND EXPRESSED IN $\mu \mathrm{M} / \mathrm{mL}$

\begin{tabular}{ccc|cc|cc}
\hline & \multicolumn{2}{c|}{$\begin{array}{c}\text { Staphyloccocus } \\
\text { aureus }\end{array}$} & \multicolumn{2}{c|}{$\begin{array}{c}\text { Escherichia } \\
\text { coli }\end{array}$} & \multicolumn{2}{c}{$\begin{array}{c}\text { Canidia } \\
\text { albicans }\end{array}$} \\
\hline Compound & MIC & MBC & MIC & MBC & MIC & MFC \\
Gallic acid & $>2000$ & $>2000$ & $>2000$ & $>2000$ & $>2000$ & $>2000$ \\
1 & 2000 & $>2000$ & 2000 & $>2000$ & 2000 & $>2000$ \\
2 & 2000 & $>2000$ & 2000 & $>2000$ & 2000 & 2000 \\
\hline
\end{tabular}

Gallic acid was used as the reference compound in 1970 from Musa sapientum ${ }^{23}$. It was also isolated from Tillandsia recurvata ${ }^{19}$, Brazil $^{24}$, Artemisia argyi ${ }^{25}$ and recently from Piptadenia gonoacantha ${ }^{26}$.

Our results provide remarkable support for the use of E. humifusa as traditional medicine, e.g. for treatment of dysentery, enteritis and hematochezia. Furthermore, E. humifusa can be considered as a new natural source of the bioactive terpenoid compounds 1-2. Understanding the chemical nature of the active principle(s), it will provide an opportunity to synthesize new and effective antimicrobial (including antitumor) drugs.

\section{Conclusion}

In this study, we have provided the experimental data to support the use of E. humifusa as a herbal medicine. Our results demonstrated that $E$. humifusa crude extracts and isolated compounds exhibited potent antimicrobial and antitumor properties. It is a good resource of bioactive compounds such as cycloart-23E-en-3 $\beta$-25-diol (1), 24-methylene cycloartanone (2), which were considered to be powerful antimicrobial agents. We do not discount the possibility of other compounds in the extracts that could also function, as antimicrobial agents, since the antimicrobial activity may be the result of a combined or synergistic effect of some undetermined compounds in the extract in this study. This plant might serve as a source of natural compounds having antimicrobial and antitumor properties, which should be further researched for microbial infections and cancer.

\section{ACKNOWLEDGEMENTS}

The authors are grateful to Dr. G.M. Shen, Xinjiang Institute of Ecology and Geography for his help with plant identification. This work was supported by the China National Funds for Distinguished Young Scientists (30925045) and the CAS/SAFEA International Partnership Program for Creative Research Teams.

\section{REFERENCES}

1. F. Sahin, M Güllüce, D. Daferera, A. Sökmen, M. Sökmen, M. Polissiou and G. Agar, H Özer, Food Cont., 15, 549 (2004).

2. C. Thetsrimuang, S. Khammuang, K. Chiablaem, C. Srisomsap and R. Sarnthima, Food Chem., 128, 634 (2011).

3. A.G. Ramchandani, R.S. Chettiyar and S.S. Pakhale, Food Chem., 119, 298 (2010).

4. P.-H. Chuang, C.-W. Lee, J.-Y. Chou, M. Murugan, B.-J. Shieh and H.-M. Chen, Bioresour. Technol., 98, 232 (2007). 
5. Y. Peng, L. Zhang, F.o Zeng and J.F. Kennedy, Carbohyd. Polym., 59, 385 (2005)

6. G. Webester, Ann. Missouri Bot. Gard., p. 33-144 (1994).

7. P.K. Singla and K. Pathak, Fitoterapia, 61, 483 (1990).

8. C. Valente, M. Pedro, A. Duarte, M.S.J. Nascimento, P.M. Abreu and M.-J.U. Ferreira, J. Nat. Prod., 67, 902 (2004).

9. S. Sutthivaiyakit, M. Thapsut and V. Prachayasittikul, Phytochemistry, 53, 947 (2000).

10. J. Hohmann, D. Rédei, P. Forgo, J. Molnár, G. Dombi and T. Zorig, J. Nat. Prod., 66, 976 (2003).

11. D. Natarajan, S.J. Britto, K. Srinivasan, N. Nagamurugan, C. Mohanasundari and G. Perumal, J. Ethnopharmacol., 102, 123 (2005).

12. M. Sudhakar, Ch.V. Rao, P.M. Rao, D.B. Raju and Y. Venkateswarlu, Fitoterapia, 77, 378 (2006).

13. Pharmacopoeia Commission of the Ministry of Public Health of PRC Pharmacopoeia of the People's Republic of China (Part 1), Medical Science Press, Beijing, p. 118 (2010).

14. T. Yoshida, Y. Amakura, Y.Z. Liu and T. Okuda, Chem. Pharm. Bull. (Tokyo), 42, 1803 (1994)
15. Z.-Q. Lu, G.-T. Chen, J.-Q. Zhang, H.-L. Huang, S.-H. Guan and D.-A. Guo, Helv. Chim. Acta, 90, 2245 (2007).

16. D. Kalemba and A. Kunicka, Curr. Med. Chem., 10, 813 (2003).

17. L. Runhui, W. Hanbo and K. Lingyi, Chinese Trad. Herbal Drugs, 32, 107 (2001)

18. Y.P. Shi and Z.J. Jia, Indian J. Chem., 36B, 1038 (1997).

19. G.M. Cabrera and A.M. Seldes, J. Nat. Prod., 58, 1920 (1995).

20. V.L.T. Hoang, Y. Li and S.-K. Kim, Bioorg. Amp.; Med. Chem. Lett., 18, 2083 (2008).

21. A.N. Starratt, Phytochemistry, 11, 293 (1972).

22. W. De-Eknamkul and B. Potduang, Phytochemistry, 62, 389 (2003).

23. F.F. Knapp and H.J. Nicholas, Steroids, 16, 329 (1970).

24. T.L.G. Lemos, P.C.L. Nogueira, J.W. Alencar and A.A. Craveiro, J. Essent. Oil Res., 7, 561 (1995).

25. A. Lao, F. Yasuo and T. Takashi, Chem. Pharm. Bull., 32, 723 (1984).

26. M.G.D. Carvalho, M.A.R. Cardozo, F.E.A. Catunda and A.G. de Carvalho, Ann. Brazilian Acad. Sci., 82, 561 (2010). 